\title{
Preparation and comparative stability of a kaolinite- tetrabutylphosphonium bromide intercalation compound for heat and solvent treatments
}

\author{
Shingo Machida ${ }^{\text {, Régis Guégan }}{ }^{b}$ and Yoshiyuki Sugahara ${ }^{a, c^{*}}$
}

aDepartment of Applied Chemistry, School of Advanced Science and Engineering, Waseda University, 3-4-1 Okubo, Shinjuku-ku, Tokyo 169-8555, Japan

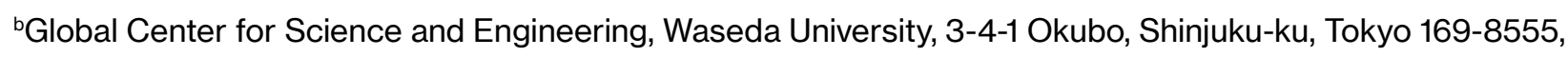
Japan

cKagami Memorial Institute for Materials Science and Technology, Waseda University, 2-8-26 Nishiwaseda, Shinjuku-ku, Tokyo 169-0051, Japan

*E-mail: ys6546@waseda.jp

Number of pages: 4

Number of figures: 5 


\section{Figures S}

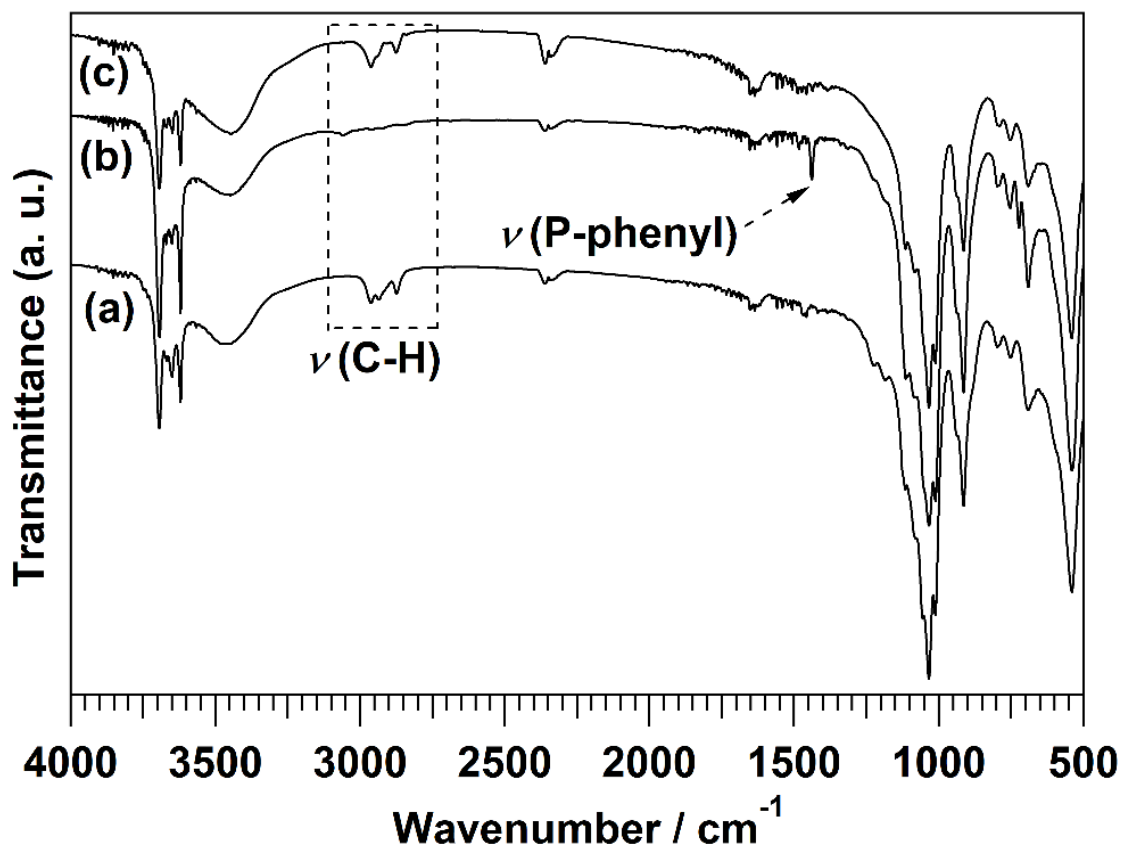

Figure S1. IR spectra in the $4000-500 \mathrm{~cm}^{-1}$ range of (a) Kaol-TBPBr, (b) Kaol-TPhPBr and (c) Kaol-TBABr.

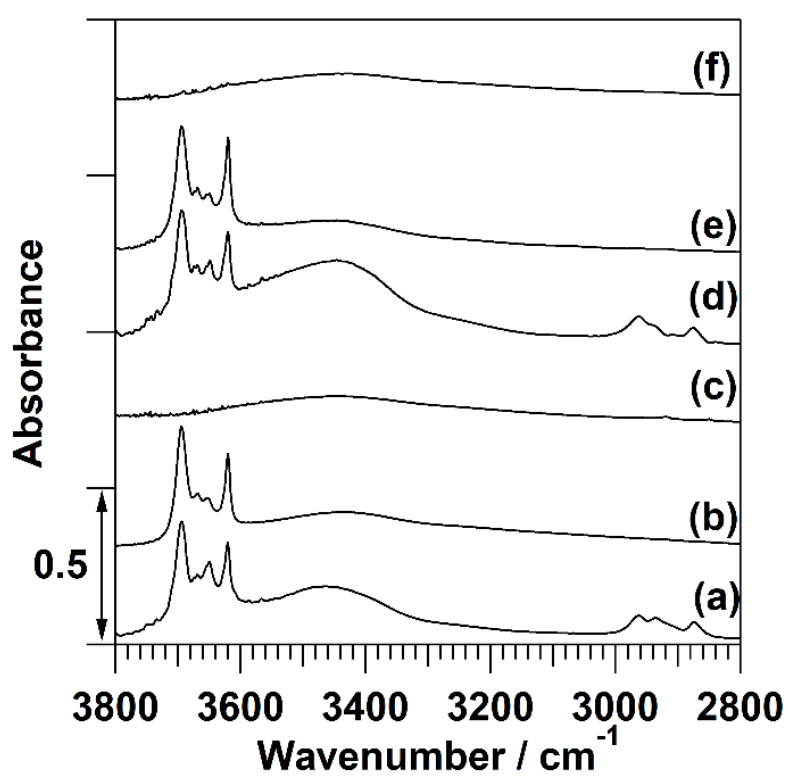

Figure S2. IR spectra in the $3800-2800 \mathrm{~cm}^{-1}$ range of (a) Kaol-TBPBr, (b) Kaol-TBPBr_400, (c) Kaol-TBPBr_800, (d) Kaol-TBABr, (e) Kaol-TBABr_350 and (f) Kaol-TBABr_800. 

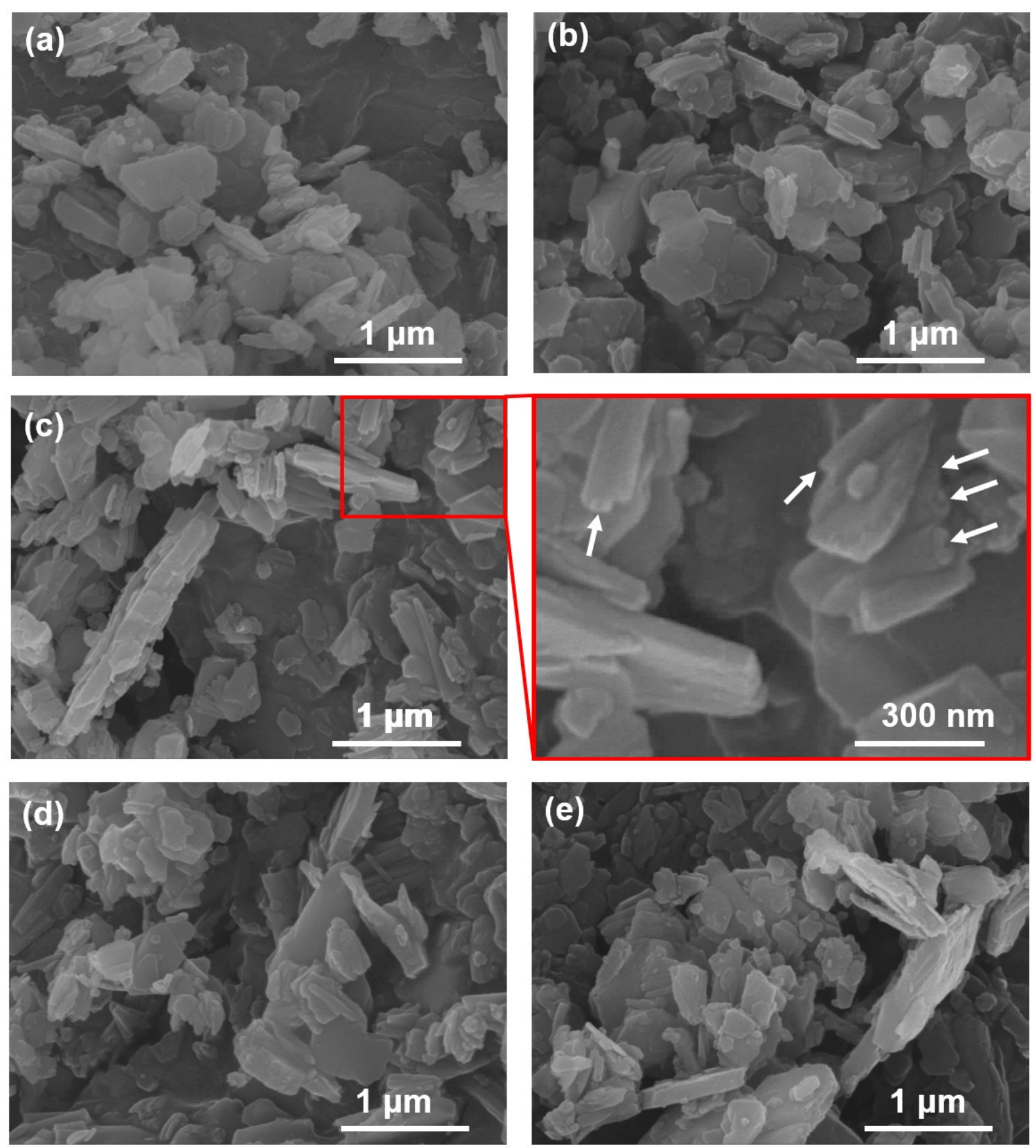

Figure S3. FE-SEM images of (a) kaolinite, (b) Kaol-DMSO, (c) Kaol-TBPBr (white arrows indicate tubes), (d) Kaol-TPhPBr and (e) Kaol-TBABr. 


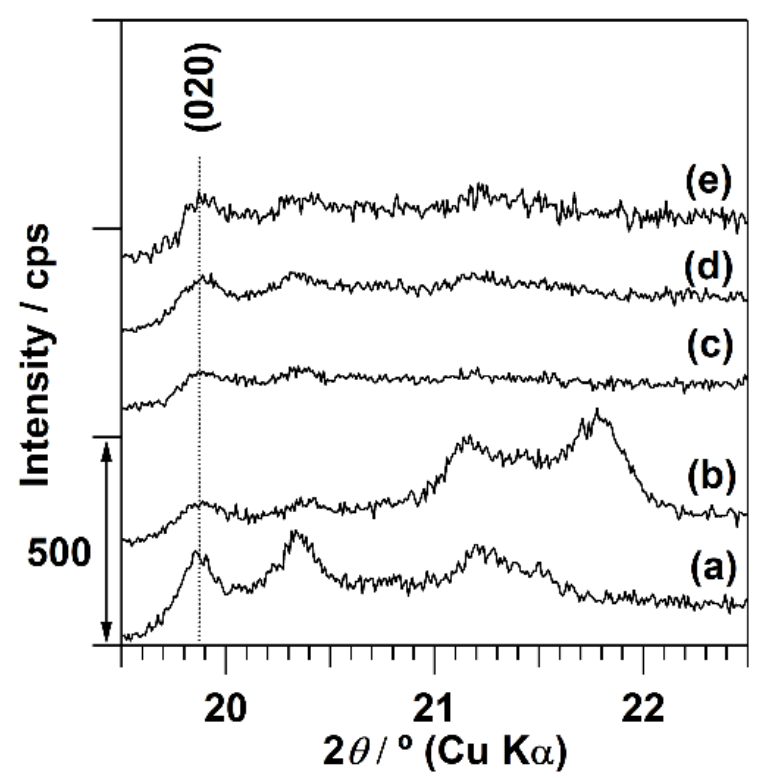

Figure S4. XRD patterns of (a) kaolinite, (b) Kaol-DMSO, (c) Kaol-TBPBr, (d) Kaol-TPhPBr and (e) Kaol-TBABr in 19.5-22.5 two $\theta$ angle region.
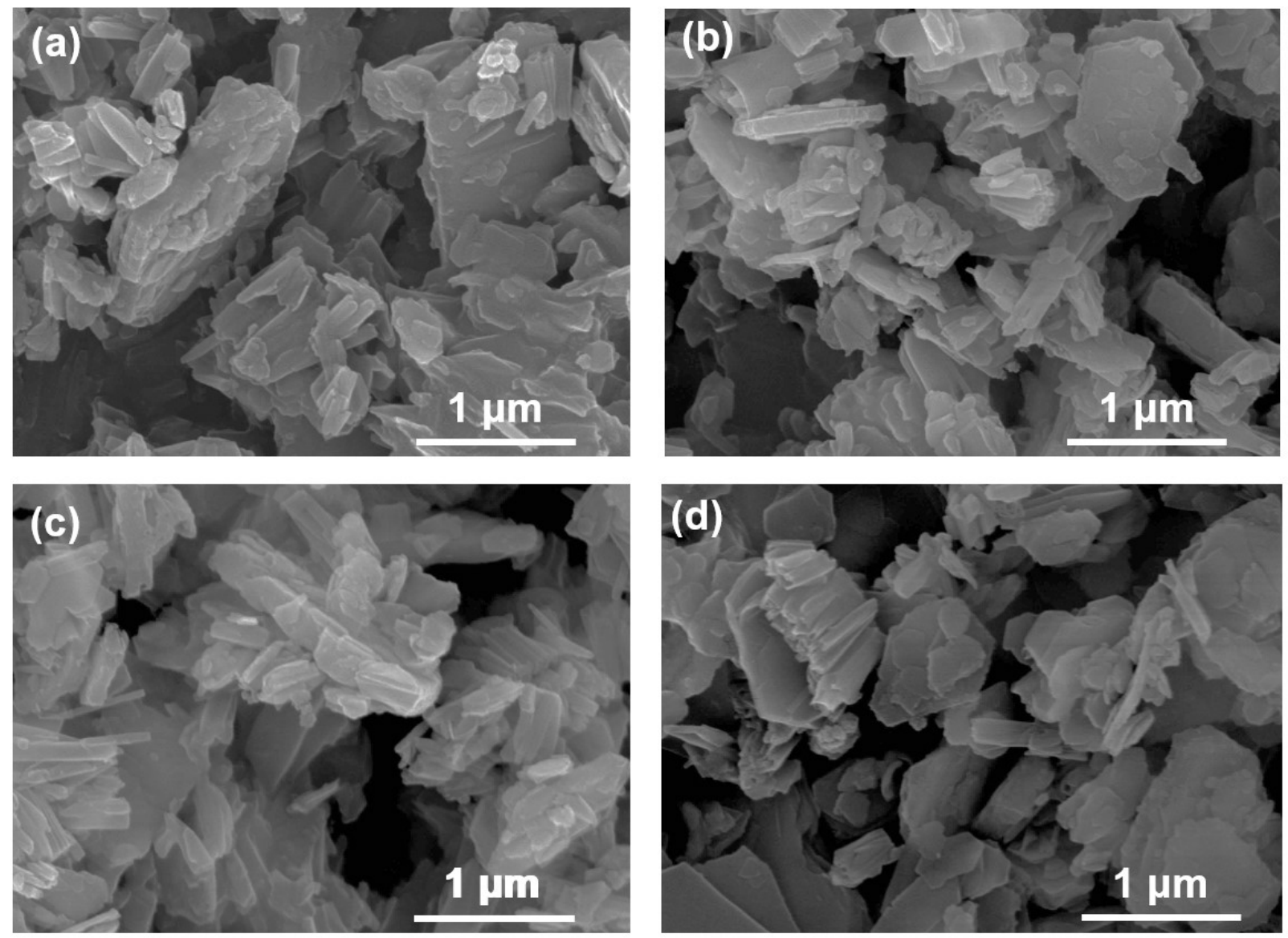

Figure S5. FE-SEM images of Kaol-TBPBr upon refluxing in (a) methanol, (b) acetone, (c) toluene and (d) ethanol. 